\title{
Home-made capillary mounting setup for shaky hands
}

\author{
B. Fournier \\ Institut Galien Paris-Saclay, CNRS UMR 8612, Université Paris-Saclay, 92296 Châtenay-Malabry, France
}

The capillary mounting of single crystals can be necessary under some circumstances prior to X-ray diffraction experiments. When crystals are air-sensitive or may undergo a desolvation, a deterioration of the samples may be observed over time which will affect the data quality during measurements. Using protective vacuum oil and working at low temperature help slowing down crystal quality decay, however capillary mounting offers a better air protection by isolating the samples [1,2]. This kind of mounting may also offer a protection of the experimenter when the crystalline compounds exhibit health hazard, it is then relevant for a better respect of hygiene and safety rules in lab workspaces.

Capillary mounting requires patience and dexterity, and so can be a matter of apprehension. Its success rate will strongly depend on the capillary size and the operator's experience. Some methods have been suggested in literature [3, 4]. However, to prepare a series of samples, the required work is time demanding and a significant fraction of crystals may be lost.

In this work, a home-made setup for capillary mounting is described. A crystal sample mounted on the top of a glass fibre can be slid in a capillary with an inner diameter smaller than $1 \mathrm{~mm}$. The capillary mounting is manually performed thanks to a micrometric translation stage and a goniometer head with five degrees of freedom $\mathrm{Rx}, \mathrm{Ry}, \mathrm{X}, \mathrm{Y}, \mathrm{Z}$. The operation is monitored using a small numerical microscope with its output displayed on computer screen by a simple program written in Python.

[1] Eich A., Schlüter S., Schnakenburg G. \& Beck J. (2013). Z. Anorg. Allg. Chem. 639, (2), 375-383

[2] Haffner A. \& Johrendt D. (2017). Z. Anorg. Allg. Chem. 643, 1717-1720

[3] Przybylska M. (1988). J. Appl. Cryst. 21, 272-273

[4] Basavappa R., Petri E. T. \& Tolbert B. S. (2003) J. Appl. Cryst. 36, 1297-1298

Keywords: single crystal, capillary mounting, home-made setup 